\title{
An assessment of the role of the centrifugal acceleration mechanism in high altitude polar cap oxygen ion outflow
}

\author{
H. Nilsson ${ }^{1}$, M. Waara ${ }^{1}$, O. Marghitu ${ }^{2,3}$, M. Yamauchi ${ }^{1}$, R. Lundin ${ }^{1}$, H. Rème ${ }^{4}$, J.-A. Sauvaud ${ }^{4}$, I. Dandouras ${ }^{4}$, \\ E. Lucek ${ }^{5}$, L. M. Kistler ${ }^{6}$, B. Klecker ${ }^{2}$, C. W. Carlson ${ }^{7}$, M. B. Bavassano-Cattaneo ${ }^{8}$, and A. Korth ${ }^{9}$ \\ ${ }^{1}$ Swedish Institute of Space Physics, Kiruna, Sweden \\ ${ }^{2}$ Max-Planck-Institut für Extraterrestriche Physik, Garching, Germany \\ ${ }^{3}$ Institute for Space Sciences, Bucharest, Romania \\ ${ }^{4}$ Centre d'Etude Spatiale des Rayonnements, Toulouse, France \\ ${ }^{5}$ Imperial College of Science, Technology and Medicine, London, UK \\ ${ }^{6}$ University of New Hampshire, Durham, USA \\ ${ }^{7}$ Space Science Laboratory, University of California, Berkeley, USA \\ ${ }^{8}$ Istituto di Fisica dello Spazio Interplanetario, Roma, Italy \\ ${ }^{9}$ Max-Planck-Institut für Sonnensystemforschung, Katlenburg-Lindau, Germany
}

Received: 15 March 2007 - Revised: 28 November 2007 - Accepted: 17 December 2007 - Published: 4 February 2008

\begin{abstract}
The role of the centrifugal acceleration mechanism for ion outflow at high altitude above the polar cap has been investigated. Magnetometer data from the four Cluster spacecraft has been used to obtain an estimate of magnetic field gradients. This is combined with ion moment data of the convection drift and the field-aligned particle velocity. Thus all spatial terms in the expression for the centrifugal acceleration are directly obtained from observations. The temporal variation of the unit vector of the magnetic field is estimated by predicting consecutive measurement-points through the use of observed estimates of the magnetic field gradients, and subtracting this from the consecutively observed value. The calculation has been performed for observations of outflowing $\mathrm{O}^{+}$beams in January to May for the years 2001-2003, and covers an altitude range of about 5 to $12 R_{E}$. The accumulated centrifugal acceleration during each orbit is compared with the observed parallel velocities to get an estimate of the relative role of the centrifugal acceleration. Finally the observed spatial terms (parallel and perpendicular) of the centrifugal acceleration are compared with the results obtained when the magnetic field data was taken from the Tsyganenko T89 model instead. It is found that the centrifugal acceleration mechanism is significant, and may explain a large fraction of the parallel velocities observed at high altitude above the polar cap. The magnetic field model results underestimate the centrifugal acceleration at the highest altitudes investigated and show some systematic differences as compared to the observations in the lower altitude ranges investigated. Our results indicate that for altitudes corresponding to magnetic field values of more than $50 \mathrm{nT}$ a test particle
\end{abstract}

Correspondence to: H. Nilsson

(hans.nilsson@irf.se) model with a steady state magnetic field model, a realistic convection model and an initial velocity of about $20 \mathrm{k} \mathrm{m} \mathrm{s}^{-1}$ at $5 R_{E}$ should be able to reproduce the main part of our observational results.

Keywords. Magnetospheric physics (Magnetosphereionosphere interactions; Polar cap phenomena) - Space plasma physics (Charged particle motion and acceleration)

\section{Introduction}

The purpose of this paper is to quantitatively assess the role of the centrifugal acceleration mechanism in high altitude polar cap ion outflow. The centrifugal acceleration mechanism accelerates charged particles along the field line due to the component of the $\boldsymbol{E} \times \boldsymbol{B}$ drift along the direction of change of the magnetic field direction. A basic introduction is given in Northrop (1963); Cladis (1986); Horwitz et al. (1994). The data set used is the same as in a previous study (Nilsson et al., 2006b), but we now include magnetic field data from all the four Cluster spacecraft. The four spacecraft data allows us to obtain estimates of the spatial and temporal variation of the magnetic field. Together with the $\boldsymbol{E} \times \boldsymbol{B}$ drift and the parallel ion velocity obtained from the Cluster CODIF ion spectrometer this allows us to estimate the centrifugal acceleration.

The polar cap ion outflow we will show and discuss in this paper emanate from the ionospheric projection of the cusp (Nilsson et al., 2004; Bogdanova et al., 2004; Bouhram et al., 2004; Dubouloz et al., 2001). The first to discuss the role of the centrifugal acceleration for polar cap ion outflow was Cladis (1986), who used model magnetic and electric fields and test particle trajectories. The strongest energization was

Published by Copernicus Publications on behalf of the European Geosciences Union. 
found in the central plasma sheet (CPS) due to the strong change of the magnetic field direction there. Particles moving along the trajectories reaching the highest altitudes above the polar cap experienced significant acceleration also before reaching the CPS, about $100 \mathrm{eV}$ energy increase $\left(35 \mathrm{~km} \mathrm{~s}^{-1}\right)$ after $2 \mathrm{~h}$ travel time. A 1-dimensional fluid calculation by Swift (1990) confirmed the effect for $\mathrm{H}^{+}$, but found that the $\mathrm{H}^{+}$population had a "smothering" effect on the $\mathrm{O}^{+}$outflow which was not much affected by the centrifugal acceleration. Later Horwitz et al. (1994) included the centrifugal acceleration term in a time-dependent, semikinetic model of polar plasma outflow, and found that the effect on the total $\mathrm{O}^{+}$ outflow was significant, especially for a cool exobase situation. Demars et al. (1996) examined the effect of the centrifugal acceleration on the polar wind using a particle-in-cell code coupled with a time-dependent, three-dimensional hydrodynamic model of the polar ionosphere. They found the effects of centrifugal acceleration to be negligible. High altitude measurements such as those we present in this paper does not directly deal with this controversy regarding the total outflow, as all observed particles have reached escape energy. Our interest lies in the further energization of escaping particles, and their role in the global circulation of mass and energy in the magnetospheric system (Winglee et al., 2002; Winglee, 2004) as well as the final fate of these ions (Ebihara et al., 2006). Delcourt (1994) found that centrifugal effects could be important near the frontside magnetopause. This is in line with the results reported by Nilsson et al. (2006b) and Arvelius et al. (2005) who made simple estimates of the centrifugal acceleration based on high altitude Cluster spacecraft data, and found it to be significant.

One of the first indications that centrifugal acceleration may be important are the observations of cold $O^{+}$beams in the tail, which have close to the same parallel velocity as simultaneously observed $\mathrm{H}^{+}$populations (Frank et al., 1977; Hirahara et al., 1996; Seki et al., 1998). A velocity filter effect may explain this similarity, in particular in the tail where both $\mathrm{H}^{+}$and $\mathrm{O}^{+}$ions may have had a significant travel distance from a common source region. However this explanation has some limitations as discussed in Nilsson et al. (2004, 2006b). One such limitation is that the velocity filter effect, if it is to create similar parallel bulk velocities for the two ion species, will also yield a narrow velocity distribution for both species, not just $\mathrm{O}^{+}$. This is not the case for our observations. Centrifugal acceleration which provides the same acceleration to all species independent of mass is another obvious candidate to explain this phenomenon. As shown by Nilsson et al. (2006b) this similarity of the parallel bulk velocity for $\mathrm{H}^{+}$and $\mathrm{O}^{+}$ions is common already above the polar cap, from about $5 R_{E}$ altitude. Ions at progressively higher altitudes are also further energized (Nilsson et al., 2004; Arvelius et al., 2005; Nilsson et al., 2006b). It is therefore of interest to study energization mechanisms in the high altitude polar cap. Acceleration mechanisms that lead to the same parallel bulk velocity are of particular in- terest. Other energization candidates that lead to the same parallel bulk velocity for $\mathrm{H}^{+}$and $\mathrm{O}^{+}$ions include acceleration in drifting density inhomogeneities (Antonova, 1983), acceleration due to the ponderomotive force (Guglielmi and Lundin, 2001) and stochastic acceleration in Alfvén waves (Chaston et al., 2004), mechanisms which essentially work by heating the particles to the same thermal velocity, which through the mirror force may lead to the same parallel bulk outflow velocity. The main importance of these mechanisms is however that they provide more energy to the heavy ions. If the heavy ions do not reach the same thermal velocity as the lighter ions, these mechanisms in combination with velocity filtering may still result in the same parallel bulk velocity. Experimentally assessing the role of the centrifugal acceleration in the polar cap ion outflow is essential to understand which mechanisms are most important under different circumstances.

The centrifugal acceleration is however of a more general importance. Its role has been discussed also for other planets. Delcourt et al. (2002) investigated the centrifugal acceleration near Mercury. Haider (1996) discussed the role of the polar wind and associated centrifugal acceleration of ions for the transport of ionospheric ions through the Martian tail. Nilsson et al. (2006a) noted the possible role of centrifugal acceleration of ions associated with the small scale magnetic anomalies of Mars acting together with the large scale solar wind electric field. For the Earth's magnetosphere the role of centrifugal acceleration has primarily been discussed in terms of substorm dynamics as in, e.g. Korth and $\mathrm{Pu}$ (2001). A direct observation of the velocity change caused by centrifugal acceleration during a substorm dipolarization event was reported by Liu et al. (1994). For such a direct observation to be possible the centrifugal acceleration must not only dominate the temporal variation of the observed parallel velocity, the velocity increase must also be significant relative to the energy resolution of the instrument. The higher the initial velocity, the higher the acceleration must be.

We will estimate the centrifugal acceleration based on the observed parameters which determine the centrifugal acceleration. An acceleration which is insignificant on a satellite spin to spin time scale ( $4 \mathrm{~s}$ for Cluster) can still be significant for the total outflow. The method employed in this paper allows for the determination also of such weaker centrifugal acceleration. We will also present a comparison of the centrifugal acceleration when the magnetic field is taken from a magnetic field model as compared to the observed magnetic field. The reason for this is to show how much of our results depend on the particular configuration of the magnetic field, and how much depends on the observed parallel and perpendicular velocities of the outflowing ions. 


\section{Theory}

The centrifugal acceleration of charged particles moving along magnetic field lines in the presence of a finite convection electric field is

$\frac{d V_{\|}}{d t}=\boldsymbol{V}_{E} \cdot \frac{d \hat{\boldsymbol{b}}}{d t}=\boldsymbol{V}_{E} \cdot\left(\frac{\partial \hat{\boldsymbol{b}}}{\partial t}+V_{\|} \frac{\partial \hat{\boldsymbol{b}}}{\partial s}+\left(\boldsymbol{V}_{E} \cdot \nabla\right) \hat{\boldsymbol{b}}\right)$

where $V_{\|}$is the field-aligned (parallel) velocity of the particle, $\boldsymbol{V}_{E}$ is the $\boldsymbol{E} \times \boldsymbol{B}$ drift (i.e. the magnetic field perpendicular drift velocity), $\mathrm{s}$ is a vector along the magnetic field direction and $\hat{b}$ is the unit vector in the direction of the magnetic field (Northrop, 1963; Cladis, 1986). The work on the particles is done by the convection electric field, and the particles will therefore experience a displacement in the direction of the electric field. The displacement is due to the component of the inertial drift along the direction of the convection electric field. The inertial drift is given by

$\boldsymbol{V}_{\text {inertial }}=\frac{v_{\|}}{\omega_{c}} \hat{\boldsymbol{b}} \times\left(\frac{\partial \hat{\boldsymbol{b}}}{\partial t}+V_{\|} \frac{\partial \hat{\boldsymbol{b}}}{\partial s}+\left(\boldsymbol{V}_{E} \cdot \nabla\right) \hat{\boldsymbol{b}}\right)$

where $\omega_{c}$ is the ion cyclotron frequency and all other variables as above. The middle term inside the brackets in this equation is the usually dominating curvature drift. The total transverse drift in the direction of the electric field a particle has experienced can conveniently be estimated from the energy equation (Cladis, 1986; Northrop, 1963).

In subsequent discussions we will refer to the three terms in the bracket on the right hand side of Eq. (1) as the temporal term (the $\frac{\partial \hat{b}}{\partial t}$ term), the parallel term (dependent on $v_{\|}$) and the perpendicular term. It is noteworthy that only the parallel component depends on the initial energy of the particle. The other terms depend on the magnitude of the $\boldsymbol{E} \times \boldsymbol{B}$ drift and the change of direction of the magnetic field, which are the same for all particles. For the parallel component, the time a particle will spend in a region with a certain acceleration is inversely proportional to its parallel velocity, so that the net acceleration per length unit along the field line will be the same for all particles, but the work done (energy increase) will be larger for particles with a high field-aligned velocity. For the other two components the acceleration is the same for all particles. Thus the force is the same for all particles of a given mass, and the energy increase per travel distance will be the same for all particles of a given mass regardless of the initial velocity.

The first order spatial gradients of the magnetic field have been estimated by combining the measurements of the Cluster magnetometers from all four spacecraft. The four spacecraft represent measurements in four spatial points for each vector component of the magnetic fields. Thus an equation can be formed (for the $\mathrm{x}$ component as an example) as:

$\frac{d B_{x}}{d x} \Delta x_{41}+\frac{d B_{x}}{d y} \Delta y_{41}+\frac{d B_{x}}{d z} \Delta z_{41}=\Delta B_{x 41}$ where $B_{x}$ is the magnetic field $\mathrm{x}$ component, $\Delta x_{41}$ indicates the difference in position between spacecraft 4 and $1\left(\boldsymbol{r}_{\mathbf{1}}-\boldsymbol{r}_{\mathbf{4}}\right)$ and $\Delta B_{x 41}$ indicates the difference in the magnetic field $\mathrm{x}$ component as measured at spacecraft 1 and $4\left(B_{x s c 1}-B_{x s c 4}\right)$. The same equation can be formed for spacecraft 2 and 3 as well (keeping spacecraft 4 as reference spacecraft). A $3 \times 3$ matrix can then be formed and the $\frac{d B_{x}}{d r}$ components can be solved for using standard inverse matrix methods, given that the four spacecraft are not coplanar. The same system of equations can then be solved for the gradients of the $B_{y}$ and $B_{z}$ components. This way of solving for the gradient of the magnetic field and its limitations are discussed in Harvey (1998).

The same set of equations is used for the spatial gradient of the unit vector of the magnetic field. The gradient of the unit vector is then used to calculate the $\frac{\partial \hat{b}}{\partial s}$ and $\left(\boldsymbol{V}_{E} \cdot \nabla\right) \hat{\boldsymbol{b}}$ terms of Eq. (1). The gradient of the magnetic field is used to calculate the expected magnetic field at the next position of spacecraft 4 , and this is compared with the measured value in an attempt to estimate the $\frac{\partial \hat{b}}{\partial t}$ term.

The general uncertainty in both moment and magnetic field estimates will not matter much for a statistical study such as this. Systematic errors may on the other hand be important. It is therefore important to note that the most important measurement property is the change of direction of the unit vector of the magnetic field direction. Therefore absolute calibration errors of the spacecraft magnetometer data is not critical, only a consistent direction error between the spacecraft could introduce significant systematic errors. There is no reason to believe that such a systematic error exists. Our experimental estimates are thus rather robust.

The next possible source of error is our use of a linear gradient. Keeping all other parameters constant, the centrifugal acceleration is linearly dependent on the change of the magnetic field direction. The value we obtain by assuming a linear gradient is then an average centrifugal acceleration in the interval between our observation points. Therefore this assumption is quite suitable for a statistical data set.

\section{Observations}

\subsection{Observational techniques}

We use data from the Cluster Ion Spectrometer (CIS) on board Cluster spacecraft 4 . The CIS instrument is described in detail in Rème et al. (2001). CIS consists of two different ion spectrometers, COmposition and DIstribution Function analyzer (CODIF) which can resolve the major magnetospheric ions and Hot Ion Analyzer (HIA) which has no mass resolution but higher angular and energy resolution. We will only present results from the CODIF instrument.

CODIF can resolve $\mathrm{H}^{+}, \mathrm{He}^{++}, \mathrm{He}^{+}$and $\mathrm{O}^{+}$through a time-of-flight technique. The detector has a field-of-view of $360^{\circ}$ orthogonal to the spin plane, divided into 16 sectors of 
$22.5^{\circ}$ each. The angular resolution is likewise $22.5^{\circ}$ in the spin plane. The energy coverage in the modes of interest to us is from $15 \mathrm{eV}$ per charge up to $38 \mathrm{keV}$ per charge in up to $30 \operatorname{logarithmically~spaced~steps~with~a~} \Delta E / E$ of 0.16 . The energy resolution limits the possibility to directly observe acceleration as a velocity increase in the time series. If the initial velocity is high the acceleration must also be high in order to be resolved.

For technical reasons we use only data from $40 \mathrm{eV}$. The actual energy of the ions is frequently somewhat higher than that as the spacecraft potential is typically in the range 5$40 \mathrm{eV}$ in the polar cap (Torkar et al., 2005). As the ion beams we report typically have significantly higher energies (hundreds of $\mathrm{eV}$ up to several $1000 \mathrm{eV}$ ) this is not a major problem. One must however be aware that our data is not representative for cases when ions below the spacecraft potential (and thus well below the CIS measurement limit) dominate, which may happen in the polar cap, e.g. Eriksson et al. (2006).

Furthermore we use data from the Cluster fluxgate magnetometers (Balogh et al., 2001).

\subsection{Data set}

The data set consists of all $\mathrm{O}^{+}$beams clearly seen in the energy spectrograms of the CODIF data for spring (January to May) of the years 2001 to 2003. This corresponds to high altitude passes over the polar cap. Most of the ion beams were observed at geocentric distances between 5 and $12 R_{E}$. The beams were identified through visual inspection of energy spectrograms. The data set is described in detail in Nilsson et al. (2006b), and an introduction to the data is also given in an earlier case study (Nilsson et al., 2004). The $\mathrm{H}^{+}$ions observed simultaneously with the $\mathrm{O}^{+}$ions are also flowing out. These are mainly magnetosheath origin ions which have mirrored in the Earth's magnetic field. We therefore classify the region as the mantle, just poleward of the cusp proper. For this study we have used ion moments from spacecraft 4 , complemented with magnetometer data with $4 \mathrm{~s}(1 \mathrm{spin})$ resolution from all the four spacecraft (Balogh et al., 2001). The time resolution of the ion data varies between 4 and $16 \mathrm{~s}$. We make no distinction between the data points in this regard in the statistical study. In the presence of intense fluxes of $\mathrm{H}^{+}$ the CODIF instrument suffers from a contamination effect, where some of the $\mathrm{H}^{+}$counts are erroneously registered as $\mathrm{O}^{+}$counts. To remove data when contamination from the $\mathrm{H}^{+}$channel significantly affects the $\mathrm{O}^{+}$channel we employ the same method as was used and described in Nilsson et al. (2006b). In brief, it is utilized that contamination occur in the same energy channels as the most intense $\mathrm{H}^{+}$fluxes, so that the energy distribution of the contamination will follow that of the original $\mathrm{H}^{+}$flux (though at much lower intensity). Velocity related moments interpreting this data as $\mathrm{O}^{+}$will yield bulk velocities one fourth of the original $\mathrm{H}^{+}$bulk velocities due to the mass difference between the two ion species. If all counts in the $\mathrm{O}^{+}$channel are due to contamination from $\mathrm{H}^{+}$the perpendicular bulk velocity of $\mathrm{O}^{+}$will therefore have precisely one fourth the value for $\mathrm{H}^{+}$. The perpendicular bulk drift velocity is by far dominated by the $\boldsymbol{E} \times \boldsymbol{B}$ drift which is the same for both species, so the occurrence of the one-fourth relation in the perpendicular bulk drift is a certain sign of significant contamination. Because there is always noise, sometimes a mixture of real $\mathrm{O}^{+}$counts and contamination and sometimes not very much $\mathrm{H}^{+}$to compare with, the following criteria have been used: the perpendicular bulk velocity ratio should either be above 0.5 or below 0.2 , or the density ratio of $\mathrm{O}^{+}$to $\mathrm{H}^{+}$should be above 0.06 . The latter two criteria have been determined from visual inspection of the distribution of strong contamination events. The criteria to allow cases with a bulk velocity ratio less than 0.2 follows from the fact that contamination events have a drift velocity ratio starting at 0.25 and gradually higher the more real $\mathrm{O}^{+}$ there is. Drift velocity ratios below 0.2 occur mainly due to noise in the $\mathrm{H}^{+}$channel when little or no $\mathrm{H}^{+}$is present. The general statistics presented later is to some extent affected by this data cleaning. Therefore we have used $\mathrm{H}^{+}$moment data for the complementary set (i.e. the data points excluded by the above criteria) to create a combined $\mathrm{H}^{+}$and $\mathrm{O}^{+}$data set. This means that we have one data set where we have used $\mathrm{H}^{+}$data whenever there were no reliable moments available from the $\mathrm{O}^{+}$data. We have also calculated the centrifugal terms for all $\mathrm{H}^{+}$data with a significant $\mathrm{H}^{+}$density (above $0.1 \mathrm{~cm}^{-3}$ ). The two latter data sets are rather similar, but it is still important not to count data points twice in the combined data set. It is also worthwhile to have a simple and straightforward definition for the $\mathrm{H}^{+}$data set, rather than defining it as complementary to the $\mathrm{O}^{+}$data set. The perpendicular and temporal terms are the same for all ions, but for the parallel term it is not necessarily straightforward to combine the two data sets. It is however a main result from our previous study (Nilsson et al., 2006b) that the $\mathrm{H}^{+}$and $\mathrm{O}^{+}$parallel bulk velocities are often close to the same in the high altitude polar cap region.

Though the parallel velocity in the equation for centrifugal acceleration (Eq. 1) is the single particle velocity, we have used the parallel bulk velocity here. As we are mostly dealing with cold ion beams this is only a minor problem, and the estimate will be a typical acceleration for the conditions at the time of observation. Finally, we have only used data for energy levels above $40 \mathrm{eV}$, to avoid some problems which are sometimes encountered at the lowest energy levels of CODIF. As mentioned in Sect. 3.1 the spacecraft potential may increase the actual minimum energy a further $5-40 \mathrm{eV}$, the higher number for less dense plasmas. 


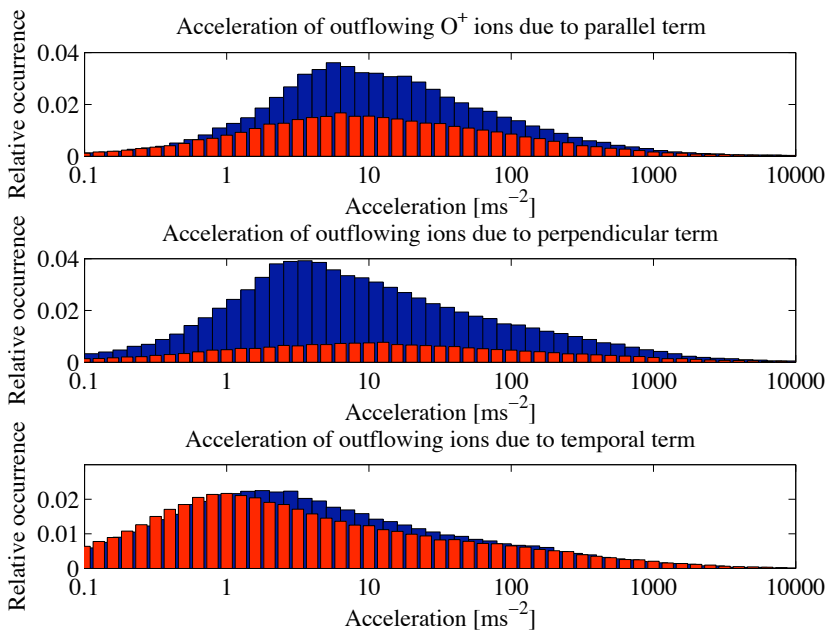

Fig. 1. Distribution of the centrifugal acceleration for all valid $\mathrm{O}^{+}$measurement points in the statistical study. The $\mathrm{x}$-axis shows the acceleration $\left[\mathrm{m} \mathrm{s}^{-2}\right]$, the $y$-axis shows the relative occurrence for positive (increased outflow velocity, blue bars) and negative acceleration (decreased outflow velocity, red bars). The red bars are shifted half a bin towards lower values to increase readability. The upper panel shows the parallel term, the middle panel the perpendicular term and the third panel the temporal term of Eq. (1).

\section{Results}

\subsection{Estimates of the centrifugal acceleration}

The three terms of the centrifugal acceleration (Eq. 1) have been estimated according to the description in Sect. 2 . The resulting distributions of observed centrifugal acceleration terms for the $\mathrm{O}^{+}$data are shown in Fig. 1. The $\mathrm{x}$-axis shows the acceleration $\left[\mathrm{m} \mathrm{s}^{-2}\right]$ and the $\mathrm{y}$-axis the relative occurrence for positive (increased outflow velocity, blue bars) and negative acceleration (decreased outflow velocity, red bars). The red bars are shifted half a bin towards lower values to increase readability when the positive and negative values are similar. The upper panel shows the parallel term, the middle panel the perpendicular term and the lower panel the temporal term of Eq. (1). As can be seen both the parallel and perpendicular terms contribute more to acceleration than to deceleration of the outflowing ions. This is particularly true for the perpendicular term, which can be understood as the centrifugal acceleration associated with a curved $E \times B$ drift path over the polar cap. This term should indeed be mainly positive for the cusp and initial polar cap region and anti-sunward convection. For the parallel term acceleration is positive for $65 \%$ and for the perpendicular term it is positive for $80 \%$ of the $\mathrm{O}^{+}$measurement points. The temporal term is essentially equally distributed between positive and negative acceleration ( $52 \%$ positive), as could be expected. To put the numbers into some context, one may note that the acceleration in $\mathrm{ms}^{-2}$ for an $\mathrm{O}^{+}$ion is almost the same as the

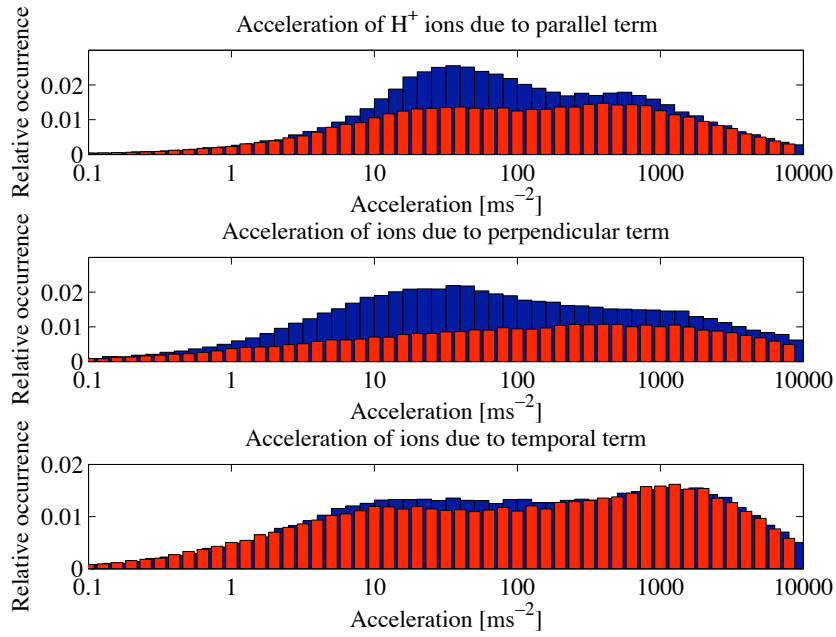

Fig. 2. Distribution of the centrifugal acceleration for all measurement points in the statistical study where the $\mathrm{H}^{+}$density was above $0.1 \mathrm{~cm}^{-3}$. The $\mathrm{x}$-axis shows the acceleration $\left[\mathrm{m} \mathrm{s}^{-2}\right]$, the $y$-axis shows the relative occurrence for positive (increased outflow velocity, blue bars) and negative acceleration (decreased outflow velocity, red bars). The red bars are shifted half a bin towards lower values to increase readability. The upper panel shows the parallel term, the middle panel the perpendicular term and the third panel the temporal term of Eq. (1).

energy increase in $\mathrm{eV}$ for a travel distance of 1 Earth radii $\left(R_{E}\right)$. More precisely the energy increase in $\mathrm{eV}$ for a $1 R_{E}$ travel distance is 1.05 times the acceleration in $\mathrm{ms}^{-2}$.

The above mentioned data exclude regions of very intense $\mathrm{H}^{+}$fluxes. We have therefore performed the same investigation for a data set based on $\mathrm{H}^{+}$data, where all measurement points with an $\mathrm{H}^{+}$density above 0.1 was used, and for a combined data set where $\mathrm{H}^{+}$data was used only when there was no valid $\mathrm{O}^{+}$data. The different terms of the centrifugal acceleration for the former data set is shown in Fig. 2.

The result is significantly different from what was obtained using the $\mathrm{O}^{+}$data. The centrifugal acceleration is distributed much more towards higher values, and negative acceleration is much more common. This is not because $\mathrm{H}^{+}$ ions as such experience a higher and more variable centrifugal acceleration, the perpendicular and temporal terms are the same for all ion species. Instead it is because significant $\mathrm{H}^{+}$fluxes are observed in or close to the cusp, close to the magnetopause and for some of our cases possibly in the magnetosheath, where the magnetic field and plasma flow appear to be more favorable for strong centrifugal acceleration.

In Fig. 3 the three terms are added together to give the total acceleration. We now use the combined data set, i.e. we use $\mathrm{O}^{+}$data, but for data points without valid $\mathrm{O}^{+}$data we use $\mathrm{H}^{+}$ data. The result looks like a fairly even mix of the $\mathrm{O}^{+}$and $\mathrm{H}^{+}$ results presented earlier. The different terms typically add up so that the amount of data points with significant acceleration is higher for the total sum than for any of the terms. 


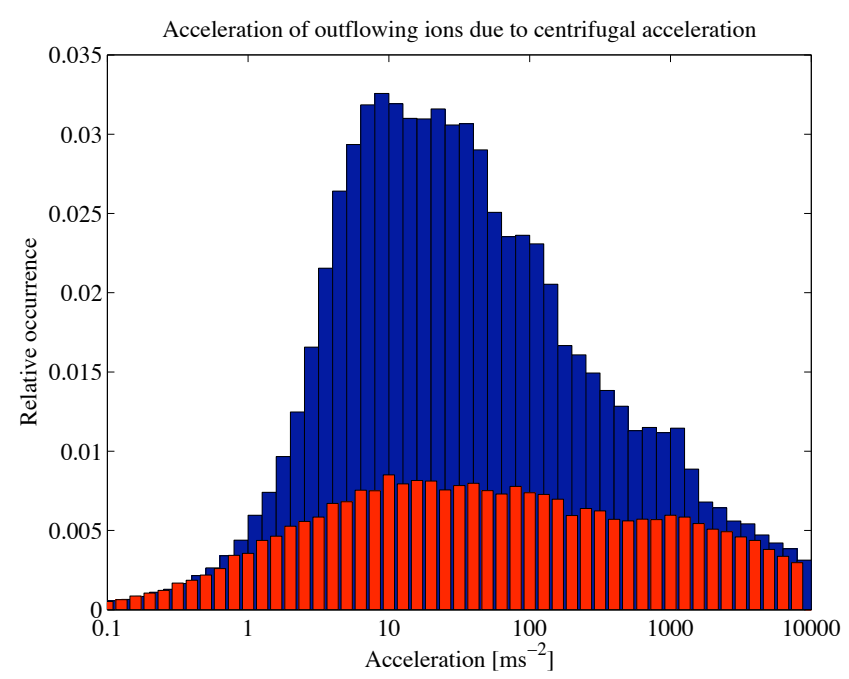

Fig. 3. Distribution of the total centrifugal acceleration for all valid measurement points in the statistical study. The combined data set was used, i.e. $\mathrm{H}^{+}$data was used when no valid $\mathrm{O}^{+}$was available. The $\mathrm{x}$-axis shows the acceleration $\left[\mathrm{m} \mathrm{s}^{-2}\right.$ ], the $\mathrm{y}$-axis shows the relative occurrence for positive (increased outflow velocity, blue bars) and negative acceleration (decreased outflow velocity, red bars). The red bars are shifted half a bin towards lower values to increase readability.

We complement the figures with Table 1 showing the percentage of data points with a positive (outward) acceleration above 10,100 and $1000 \mathrm{~ms}^{-2}$. In Table 1 we also show the same percentages for the measurement points where the $\mathrm{H}^{+}$ density was above $0.1 \mathrm{~cm}^{-3}$, and for the combined data set where $\mathrm{H}^{+}$data was used whenever the $\mathrm{O}^{+}$data was not valid because of contamination from strong $\mathrm{H}^{+}$fluxes. Note that the percentages given in Table 1 are the fraction of all data points, also including negative acceleration.

\subsection{Magnetic activity dependence}

In a study of the distribution of centrifugal acceleration it is of interest to compare to the background conditions, such as solar wind conditions and magnetic activity (e.g. the $K_{p}$ index). Relating to solar wind conditions is quite complex as one needs to take into account the time delay both from the observing satellite to the Earth's magnetosphere, as well as delays within the magnetosphere. We have therefore opted to compare the combined $\mathrm{O}^{+}$and $\mathrm{H}^{+}$data set with the $K_{p}$ index. The result is shown in Fig. 4. As can be seen there is a bimodal distribution of the acceleration for the highest $K_{p}$ interval. The low positive acceleration distribution shows a rather sharp peak at $25 \mathrm{~ms}^{-2}$ which results from the parallel term (the individual terms are not shown in the paper). For accelerations above about $100 \mathrm{~ms}^{-2}$ there are approximately the same amount of positive and negative acceleration and the distribution is very flat with no distinct peak. This is

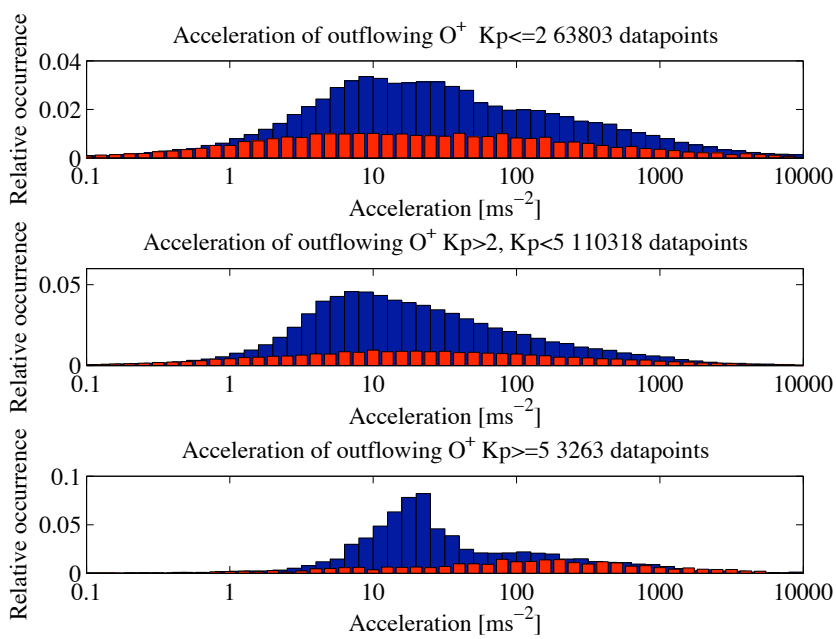

Fig. 4. Distribution of the total centrifugal acceleration for all valid measurement points in the statistical study, for three different $K_{p}$ intervals. The $\mathrm{x}$-axis shows the acceleration $\left[\mathrm{m}^{-2}\right]$, the $\mathrm{y}$-axis shows the relative occurrence for positive (increased outflow velocity, blue bars) and negative acceleration (decreased outflow velocity, red bars). The red bars are shifted half a bin towards lower values to increase readability. The upper panel shows the results for $K_{p}<=2$, the middle panel shows the results for $K_{p}>2, K_{p}<5$ and the lower panel shows the result for $K_{p}>=5$. The number of data points included in each interval is given in the text above each panel.

mainly due to the temporal term (not shown). There is remarkably little influence on the acceleration from the $K_{p}$ index, especially below $K_{p} 5$. For the highest Kp index there is a shift towards higher magnitude of the acceleration in the distribution, and a clear lack of very low acceleration values. To further check this result we also calculated the distribution of the convection velocity for the same $K_{p}$ intervals. This indeed showed an increased occurrence of high values for higher $K_{p}$ as could be expected (not shown).

We complement the $K_{p}$ dependence study with a study on the dependence of the centrifugal acceleration on observed convection velocity and the magnetic field strength. The result for the comparison with magnetic field strength is shown in Fig. 5. We have here used the $\mathrm{O}^{+}$data set as that is our main interest, but the same type of plot for the combined data set looks very similar.

The figure shows the distribution of the total centrifugal acceleration (positive in direction of outflow) $\left[\mathrm{m} \mathrm{s}^{-2}\right]$ for each interval of magnetic field strength [nT]. Each column is normalized, the sum of all data bins in a column is $100 \%$ if all data points are contained within the plot. As can be seen the more significant centrifugal acceleration occurs for low magnetic field values (high altitude). This is consistent both with higher convection velocities for low magnetic field strength, as well as more curved magnetic field lines. Also shown is a red line proportional to one over the square root of B. If the entire change with altitude was due to the higher 
Table 1. Tabulation of the percentage of positive (outward) centrifugal acceleration above the value indicated in the header. The percentage indicated is the fraction of all data points, including negative acceleration.

\begin{tabular}{|c|c|c|c|c|}
\hline Term & $\%$ positive & $\%$ above $10 \mathrm{~ms}^{-2}$ & $\%$ above $100 \mathrm{~ms}^{-2}$ & $\%$ above $1 \mathrm{kms}^{-2}$ \\
\hline Parallel $\mathrm{O}^{+}$ & 65 & 29 & 7.0 & 0.8 \\
\hline Perpendicular $\mathrm{O}^{+}$ & 80 & 31 & 10 & 1.4 \\
\hline Temporal $\mathrm{O}^{+}$ & 52 & 14 & 4.3 & 0.5 \\
\hline Total $\mathrm{O}^{+}$ & 75 & 43 & 14 & 2.0 \\
\hline Parallel $\mathrm{H}^{+}$ & 58 & 46 & 24 & 6.4 \\
\hline Perpendicular $\mathrm{H}^{+}$ & 65 & 45 & 25 & 9.2 \\
\hline Temporal $\mathrm{H}^{+}$ & 51 & 37 & 24 & 10 \\
\hline Total $\mathrm{H}^{+}$ & 62 & 51 & 30 & 13 \\
\hline Parallel combined & 63 & 34 & 12 & 2.2 \\
\hline Perpendicular combined & 78 & 38 & 15 & 3.5 \\
\hline Temporal combined & 51 & 18 & 8.6 & 3.1 \\
\hline Total combined & 73 & 47 & 19 & 4.9 \\
\hline
\end{tabular}

convection velocity at higher altitude, it could be expected that the increase would follow this curve. This is not the case. The centrifugal acceleration increases more for low magnetic field values than just due to the increased convection velocity. This was further confirmed by a similar comparison of the distribution of the centrifugal acceleration for different convection velocities (not shown).

\subsection{Comparison with a magnetic field model}

To give an indication of how close a realistic model of the centrifugal acceleration would come to our observed values, we have used the observed parallel and perpendicular bulk velocities and taken the magnetic field data from a magnetic field model. We have taken the Tsyganenko 89 model (Tsyganenko, 1989), to be able to use the $K_{p}$ index as an input parameter. In Fig. 6 we show the distribution of the ratio of the observed and model perpendicular terms for each interval of magnetic field intensity. We have used the $\mathrm{O}^{+}$data set for this comparison.

For magnetic field values down to about $50 \mathrm{nT}$ there is very little scatter and a clear tendency for the model to overestimate the centrifugal acceleration. The occurrence peak is in the bin covering an observed to model ratio of 74 to $86 \%$. Though it is a systematic difference it must be considered fairly small. At lower magnetic field values the observed centrifugal acceleration is as a general tendency higher than predicted by the model, with large scatter. The colour plot over the chosen ratio interval of 0.1 up to 10 does not show this very clearly, but the median line (red line in Fig. 6) shows how the observed value is typically several times larger than the model value.

The same plot for the parallel term is shown in Fig. 7.

The parallel term shows more variable systematic differences between the observed and model values of the centrifugal acceleration. For magnetic field values down to $50 \mathrm{nT}$,

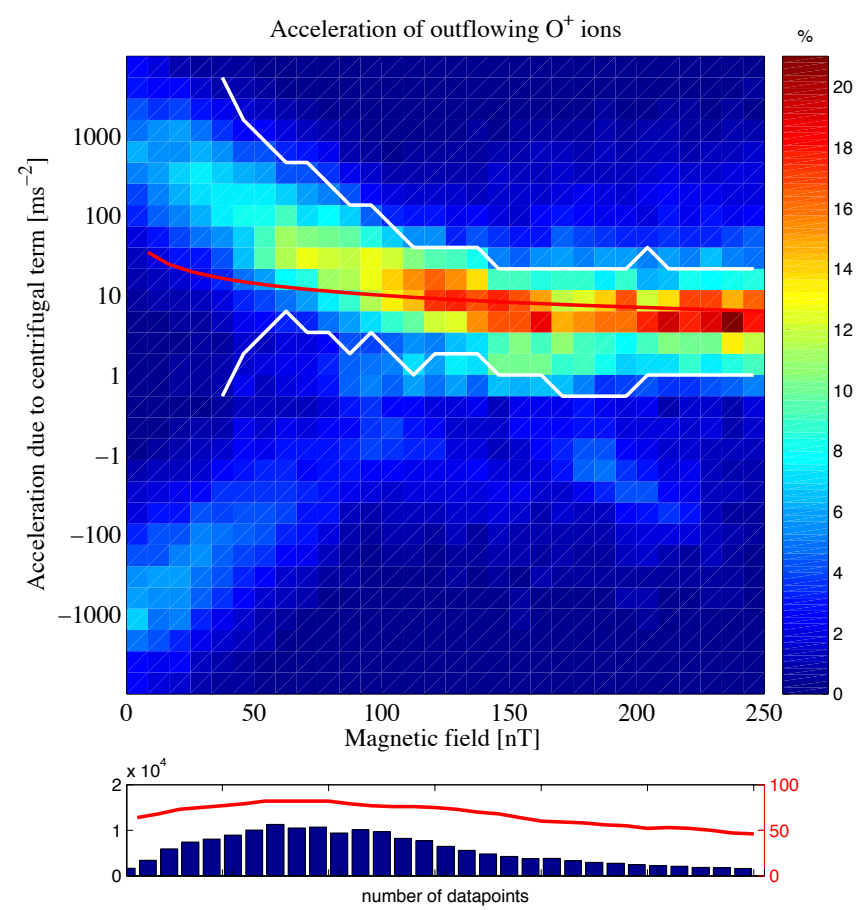

Fig. 5. Upper panel: Distribution of total centrifugal acceleration (positive in direction of outflow) $\left[\mathrm{m} \mathrm{s}^{-2}\right]$ for each interval of magnetic field strength [nT]. Each column is normalized, the sum of all data bins in a column is $100 \%$ if all data is within the plotted interval. White lines indicate the narrowest region around the maximum in each column which contains $50 \%$ of all data points. Also shown is a red line proportional to one over the square root of B. Lower panel: Number of data points contributing to each column (blue bars, left y-axis), and the number of measurement days contributing to the column (red line, right y-axis). 


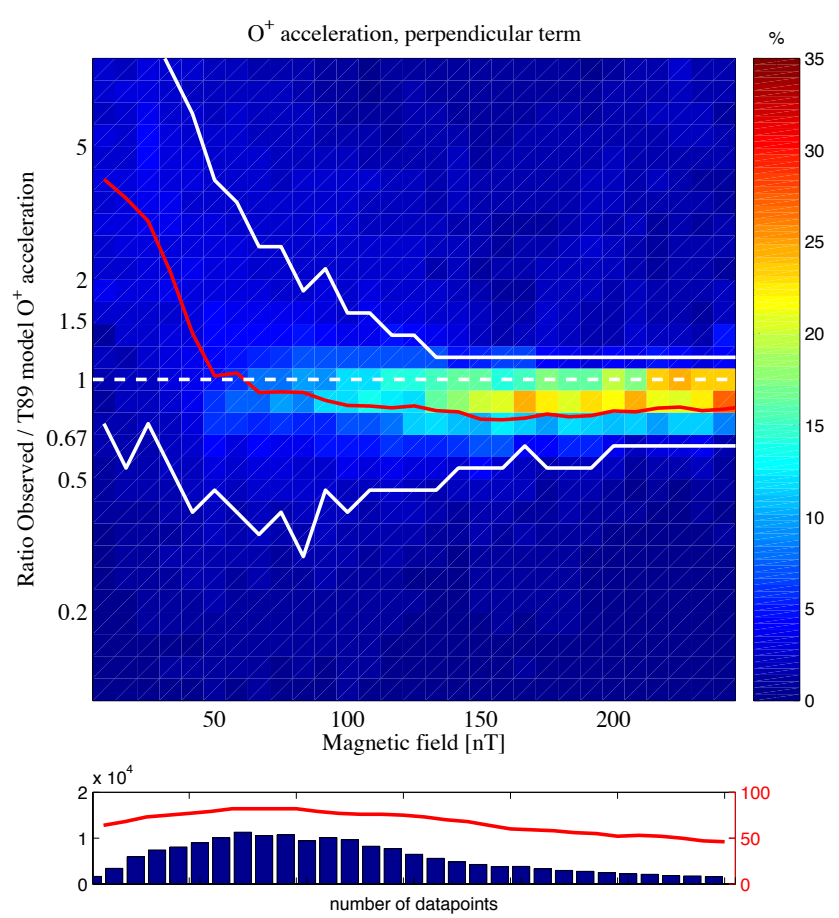

Fig. 6. Upper panel: Distribution of the ratio of the perpendicular term of the centrifugal acceleration of $\mathrm{O}^{+}$(positive in direction of outflow) from observed magnetic field values and with magnetic field values from the Tsyganenko 89 model, for each interval of magnetic field strength [nT]. Each column is normalized, the sum of all data bins in a column is $100 \%$ if all data points are contained in the plotted region. White lines indicate the narrowest region around the maximum in each column which contains $50 \%$ of all data points. A red line shows the median value of the parallel velocity ratio for each magnetic field interval. Lower panel: Number of data points contributing to each column (blue bars, left y-axis), and the number of measurement days contributing to the column (red line, right yaxis).

the model values increase systematically relative to the observed values, from a ratio of 1.5 at $250 \mathrm{nT}$ to a ratio of 0.5 at $50 \mathrm{nT}$. For magnetic field values below $50 \mathrm{nT}$ the situation is the same as for the perpendicular term, the observed values are typically higher than the model values and the scatter is large.

\subsection{Comparison with observed velocities}

So far we have presented observations of the centrifugal acceleration based on measurements of the magnetic field and the bulk drift velocities of the plasma. If the centrifugal acceleration dominates, it is plausible that we could at times observe a change of the parallel velocity with time which equals the estimated centrifugal acceleration (see for example Liu et al. (1994) cited in the introduction). However, according to our results the centrifugal acceleration only seldom reaches values above $1 \mathrm{kms}^{-2}$, which would correspond to a

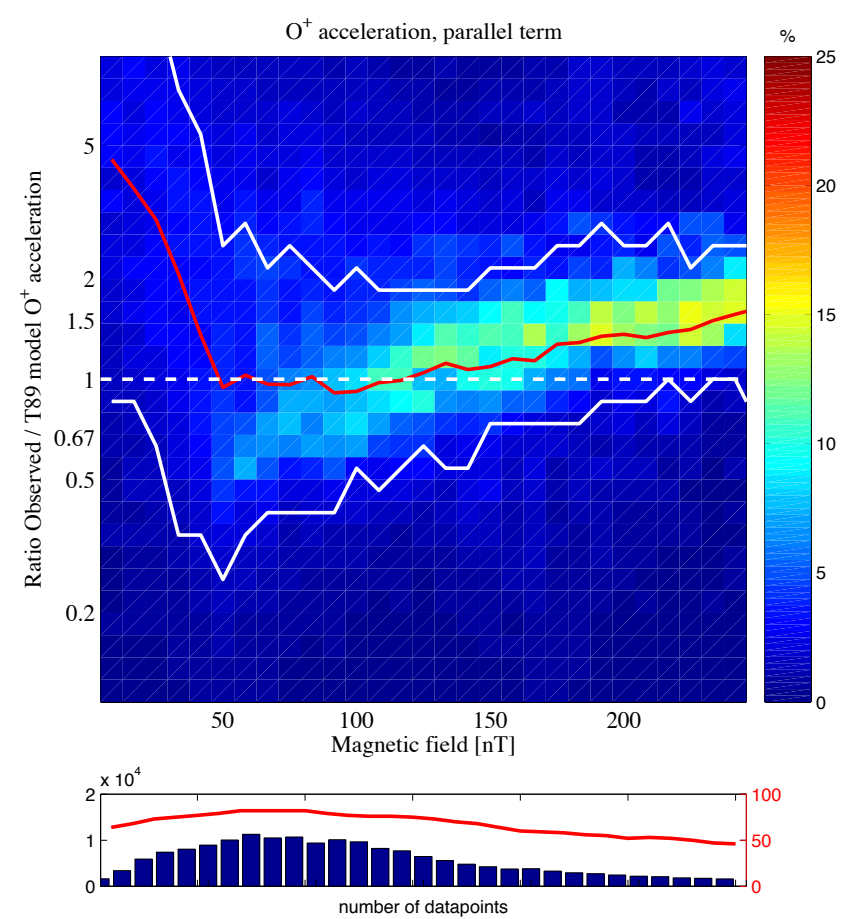

Fig. 7. Upper panel: Distribution of the ratio of the parallel term of the centrifugal acceleration of $\mathrm{O}^{+}$(positive in direction of outflow) from observed magnetic field values and with magnetic field values from the Tsyganenko 89 model, for each interval of magnetic field strength [nT]. Each column is normalized, the sum of all data bins in a column is $100 \%$ if all data points are contained in the plotted region. White lines indicate the narrowest region around the maximum in each column which contains $50 \%$ of all data points. A red line shows the median value of the parallel velocity ratio for each magnetic field interval. Lower panel: Number of data points contributing to each column (blue bars, left y-axis), and the number of measurement days contributing to the column (red line, right y-axis).

$4 \mathrm{kms}^{-1}$ velocity increase over one satellite spin. This is typically below the general variability of the observed parallel bulk velocity. The importance of the centrifugal acceleration lies rather in the total effect obtained for outflow over several $R_{E}$ distance.

A rough comparison between observed parallel velocities and the observed centrifugal acceleration can be made, by summing up the observed acceleration for each orbit, from the lowest to the highest altitude. This will show if the observed acceleration for a given orbit can approximately reproduce the observed parallel velocities. This approach ignores both temporal and latitudinal changes but should still give an approximate similarity if all acceleration is due to the centrifugal acceleration. The total parallel acceleration after a certain travel distance will depend on the parallel velocity for the perpendicular and temporal terms. This is because the time spent in a region with a particular acceleration is 


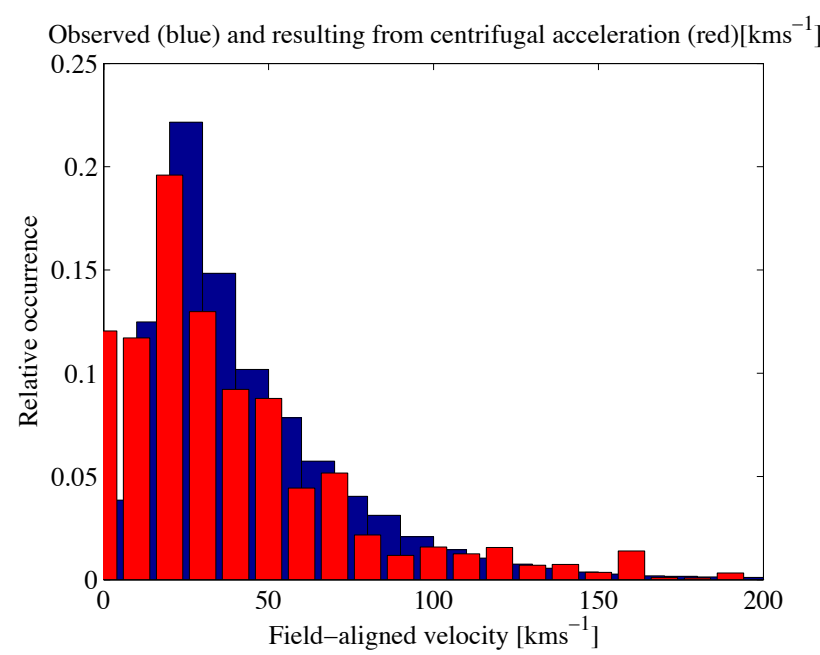

Fig. 8. Distribution of the observed parallel velocities (blue bars) and the parallel velocity resulting from the cumulative centrifugal acceleration observed at all altitudes below each observation point (red bars) $\left[\mathrm{km} \mathrm{s}^{-1}\right]$, The initial velocity at the lowest altitude of the data set for each orbit has been added to make the two distributions comparable. The y-axis shows the relative occurrence. The red bars are shifted half a bin towards lower values to increase readability.

inversely proportional to the parallel velocity. The parallel term on the other hand is proportional to the parallel velocity, so for the acceleration per travelled distance the two dependencies on the parallel velocity will cancel each other.

Such an estimate of the total velocity increase due to the centrifugal acceleration makes most sense for the $\mathrm{O}^{+}$distribution which start as a cold low energy beam at low altitude, so we have used the $\mathrm{O}^{+}$data set. The parallel velocity resulting from the centrifugal acceleration observed below each observation point has been calculated for each orbit. The initial parallel velocity at the lowest altitude in the data set of each orbit has been added to get the resulting parallel velocity. The actually observed parallel velocity was used to determine for how long time the particles experienced the calculated centrifugal acceleration. The result is shown in Fig. 8, together with the distribution of the observed parallel velocity. It is clear from Fig. 8 that the observed centrifugal acceleration, added to the initial velocity observed at the lowest altitude, have a very similar distribution to that of the observed velocities. The median initial velocity was $20 \mathrm{~km} \mathrm{~s}^{-1}$, with an average of $27 \mathrm{~km} \mathrm{~s}^{-1}$. A suitable initial velocity at 5 $R_{E}$ to use in a model would be $20 \mathrm{~km} \mathrm{~s}^{-1}$.

\subsection{Inertial drift of the particles}

The centrifugal acceleration is associated with the inertial drift of the particles in the direction of the convection electric field. In simulations such as those by Cladis (1986); Horwitz et al. (1994) this drift was ignored because it was too small to significantly affect the results. As the drift represents a non-

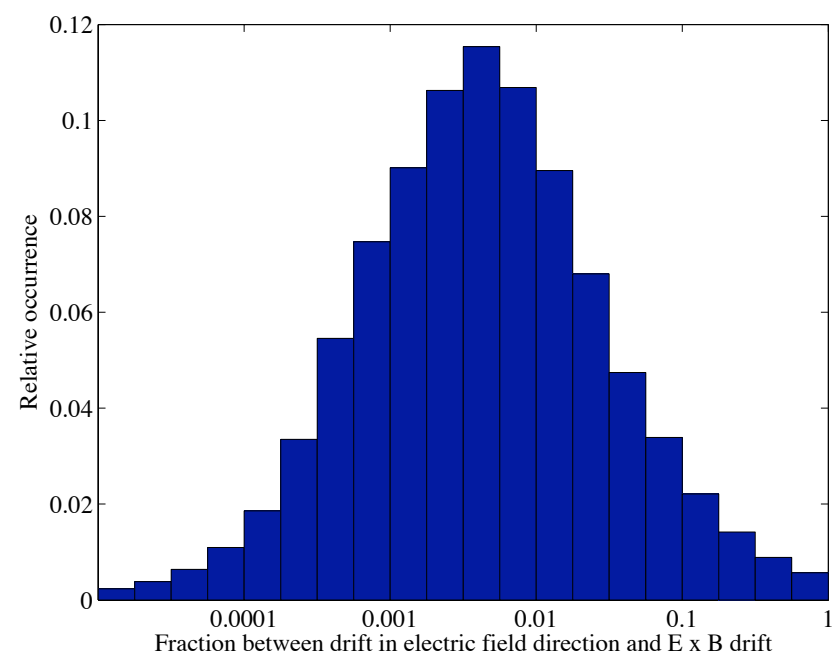

Fig. 9. Distribution of the inertial drift in the direction of the electric field direction as a fraction of the $\boldsymbol{E} \times \boldsymbol{B}$ drift velocity. The $\mathrm{y}$-axis shows the relative occurrence.

MHD behavior of the particles it is however of considerable general interest to assess quantitatively how large it is. We have therefore calculated the inertial drift along the electric field. As it is mass dependent we have used the $\mathrm{O}^{+}$data set. The distribution of this drift as a fraction of the $\boldsymbol{E} \times \boldsymbol{B}$ drift is shown in Fig. 9.

For $29 \%$ of the measurement points the inertial drift exceeds $1 \%$ of the $\boldsymbol{E} \times \boldsymbol{B}$ drift, and for $11 \%$ of the measurement points it exceeds $10 \%$. Therefore the drift will usually not be significant from a large scale plasma transport point of view. For smaller scale features it may be important.

\section{Discussion}

5.1 Role of the centrifugal acceleration in magnetospheric dynamics

The results shown in this study indicate that centrifugal acceleration is often a significant energization mechanism for outflowing $\mathrm{O}^{+}$ions at high altitude above the polar cap. For almost half of the measurement points the centrifugal acceleration is $10 \mathrm{~ms}^{-2}$ or more ( $43 \%$ for the $O^{+}$data set, $47 \%$ for the combined data set and $51 \%$ for the $\mathrm{H}^{+}$data set). This is significantly more than gravity at these altitudes of about $5 R_{E}$ and above. If such a net acceleration persists along the flight path of an oxygen ion, it will gain more than $10 \mathrm{eV}$ per $R_{E}$ traveling along the field-line. Increasing the importance of the centrifugal acceleration mechanism in the global dynamics of the magnetosphere is the fact that strong centrifugal acceleration is much more common at high altitudes / low magnetic field values (see Fig. 5). At altitudes corresponding to magnetic field values of $50 \mathrm{nT}$ the acceleration is typically $100 \mathrm{~ms}^{-2}$ (see Fig. 5). At even higher altitudes/lower 
magnetic field values the magnitude of the centrifugal acceleration increases even more, but the sign is variable, deceleration is almost equally common.

The best model to compare with appears to be Delcourt (1994), who presented simulations of centrifugal acceleration close to the frontside magnetopause. Delcourt (1994) found that the perpendicular term was more important than the parallel term which is in accordance with our findings, though the difference is not very big (see Table 1). The magnitude of the acceleration when it was significant was of the order of $10 \mathrm{~ms}^{-2}$. This is close the median of our combined data set. In Table 1 it can be seen that $47 \%$ of the total acceleration is above $10 \mathrm{~ms}^{-1}$. Significantly higher values are rather common in our data. Including a more refined magnetic field model and a temporal term in the model should, according to our result, yield even more energization from the centrifugal acceleration mechanism. Insignificant centrifugal acceleration appears much less common in our observations than in the Delcourt (1994) model. This may be due to the fact that we present data only when clear $\mathrm{O}^{+}$ion beams were present. Conditions when the $\mathrm{O}^{+}$ions were never accelerated into the instrument measurement range (see Sect. 3.1) or the fluxes were too low are not included in our data set. The initial data set used in this study consisted of 130 intervals, taken from January to May for three years, i.e. about 190 orbits. The results presented here are thus fairly typical, but may not be representative for all conditions.

Our rough estimate of the total parallel bulk velocity resulting from the observed centrifugal acceleration, shown in Fig. 8, was rather close to the observed values. This indicates that centrifugal acceleration is a major acceleration mechanism in this part of the magnetosphere.

The results presented here also indicate that in $2 \%\left(\mathrm{O}^{+}\right.$ data set) to $13 \%\left(\mathrm{H}^{+}\right.$data set $)$of the measurement points an acceleration of $1 \mathrm{~km} \mathrm{~s}^{-2}$ or above can occur. Such fast accelerations can be important in magnetospheric dynamics as discussed for example in Korth and Pu (2001); Daglis and Axford (1996). Such strong acceleration is more likely in the tail where the magnetic field curvature is expected to be stronger. Our results indicates that significant energization often occur already before the particles reach the tail.

\subsection{Equal parallel bulk velocity of $\mathrm{H}^{+}$and $\mathrm{O}^{+}$}

One interesting aspect of the centrifugal acceleration is that (for a given parallel velocity) the acceleration is the same regardless of particle mass. Therefore heavier ions will gain significantly more energy, at least for the perpendicular and temporal terms which are not dependent on the initial energy of the particle. For the parallel term a faster particle will gain more energy. The net velocity gain after traveling through a region with constant centrifugal acceleration will however be the same as for a slower particle, as the time spent in the acceleration region is as much shorter as the acceleration is stronger. Therefore the centrifugal acceleration gives a plausible explanation for why the observed parallel velocities of $\mathrm{H}^{+}$and $\mathrm{O}^{+}$at high altitude above the polar cap are close to the same most of the time (Nilsson et al., 2006b). The results presented here show that the centrifugal acceleration is indeed strong enough to contribute to this similarity. As discussed in Nilsson et al. (2006b), the centrifugal acceleration mechanism cannot explain all features of the observed $\mathrm{O}^{+}$ outflow: (1) why the similarity between the parallel bulk velocities is closest when the $\mathrm{H}^{+}$bulk velocity is calculated for the complete distribution function, not only for upward moving particles, (2) why there appears to be a coupling between the perpendicular temperatures of the two ion species, nor (3) why the perpendicular temperature of $\mathrm{O}^{+}$increases with altitude despite the action of the mirror force. The former indicates a two-stream interaction, the latter two points indicate heating (wave-particle) interaction. We cannot rule out any of these other mechanisms, nor any other of the mechanisms mentioned in the introduction and in Nilsson et al. (2006b) based on the fact that the centrifugal acceleration is significant. Studies assessing the role of these other mechanisms must also be performed in order to fully explain the similarity of the velocity of $\mathrm{H}^{+}$and $\mathrm{O}^{+}$in the high altitude polar cap.

\subsection{Modeling centrifugal acceleration}

In order to model the centrifugal acceleration the modeler needs realistic background magnetic and electric field models. This is enough to model the centrifugal acceleration itself, as it is a single particle effect. Plasma effects may be needed to correctly describe the configuration of the magnetic field in certain regions. We compared the results obtained when using an average model magnetic field instead of the observations. For magnetic field values above $100 \mathrm{nT}$ the agreement was usually quite good but with some systematic differences. Combining this result with the result shown in Fig. 5 we see that this typically corresponds to a centrifugal acceleration of about $10 \mathrm{~ms}^{-2}$. This is rather close to the typical acceleration in Delcourt (1994). At higher altitudes (magnetic field values below $100 \mathrm{nT}$ ) the resulting acceleration was often underestimated by the model. This can be expected as it represents an average field, and the more bent field-line configurations that may temporarily occur can be expected to be smoothed out in an average model. Temporal effects were of course not included in the static (but $K_{p}$ dependent) model we used. Except for the stronger accelerations observed at the highest altitudes it should be possible to reproduce the results reported here with a simple test particle model, using a realistic statistical magnetic field and convection model.

The median initial velocity, observed at the lowest altitude for each orbit where ion beams were observed, was $20 \mathrm{~km} \mathrm{~s}^{-1}$. This is thus a suitable value to use at a lower boundary of about $5 R_{E}$ if an attempt is made to model only 
the high altitude acceleration. It is also a benchmark value for models starting from lower altitudes.

\section{$5.4 \quad K_{p}$ dependence}

According to our results the $K_{p}$ index does not strongly order the centrifugal acceleration data. There are more cases of high magnitude centrifugal acceleration for high $K_{p}$, but acceleration and deceleration are approximately equally common. The temporal term increased most with $K_{p}$. In a previous study Arvelius et al. (2005) found, based on essentially the same data set as in our study, that energization at altitudes above $7 R_{E}$ was more prominent for high $K_{p}$. The acceleration at higher altitude often show deceleration as well, see Fig. 5, where the presence of significant negative acceleration can be seen. With a high magnitude, but with variable sign, the centrifugal acceleration will still lead to more occasions of high energy of the particles (extending the width of the distribution of the particles). Therefore the centrifugal acceleration can contribute to enhanced ion energization at the highest altitudes for high $K_{p}$ periods.

\subsection{The drift along the electric field direction}

The drift the particles experience along the electric field direction is typically a negligible amount of the $\boldsymbol{E} \times \boldsymbol{B}$ drift at the same time as it represents a significant energization of the particles. May inertial drifts of $1-10 \%$ of the convection velocity be significant in some circumstances? It is not likely to give measurable asymmetries in the global distribution of $\mathrm{O}^{+}$ ions itself. It may however give rise to observable small scale features. An example is a separation of small scale $\mathrm{H}^{+}$and $\mathrm{O}^{+}$structures which were initially co-located. This would happen because the inertial drift is mass dependent and thus much more significant for $\mathrm{O}^{+}$. After $1 R_{E}$ convection drift over the polar cap the displacement of the $\mathrm{O}^{+}$ions in the direction of the electric field would be 60 to $600 \mathrm{~km}$ if the inertial drift was $1-10 \%$ of the convection drift. The $\mathrm{H}^{+}$ions would have drifted 4 to $40 \mathrm{~km}$, which is negligible compared to the $\mathrm{O}^{+}$drift. For structures where differences in location of a few tens to e few hundred kilometers can be resolved the inertial drift would matter.

We have also investigated if this mechanism could be an explanation of the so called "overlapping cusp injections" observed for example by the Freja satellite (Norberg et al., 1994; Yamauchi and Lundin, 2001; Nilsson et al., 1996). The overlapping injections are interesting, because if they are caused by processes at the magnetopause they are very difficult to explain using standard reconnection theories of the solar wind magnetosphere interaction (Yamauchi and Lundin, 2001). The overlaps occur in connection to transient magnetosheath ion injections in the cusp. The overlap forms when faster ions from a newer injection overtake slower ions from an older injection. In an ideal MHD description the particles from different injections are not expected to occur on the same field line, and the overlap should not occur. Only a slight deviation of ideal MHD is needed to produce the overlaps which are typically not of very large spatial dimensions. A significant inertial drift could possibly provide this deviation from ideal MHD, something which has, as far as we are aware, never been suggested before. A first estimate of the possible significance of the inertial drift for the overlapping injections can be made as follows: Let us first assume that particles from an older injection did not experience much inertial drift, so that we need just calculate the high altitude inertial drift of the subsequent injection. From equations 1 and 2 we have that the inertial drift along the electric field is $\omega_{c}^{-1} V_{\|} / V_{\perp} a$ where a is the centrifugal acceleration which can be $10 \mathrm{~km} \mathrm{~s}^{-2}$, the gyro frequency for protons $\omega_{c}$ is about 10 at high altitude, and the ratio between parallel and perpendicular velocity may be around 10 for most conditions. We thus get an order of magnitude of $10 \mathrm{~km} \mathrm{~s}^{-1}$ for a fairly large high altitude proton inertial drift. This may at first seem significant as the convection velocity is often in the range 10 to $100 \mathrm{~km} \mathrm{~s}^{-1}$ at these altitudes. However if this inertial drift occur only in the high altitude part of the field line, it is difficult to see how it could persist for more than about $100 \mathrm{~s}$, which would yield a high altitude drift distance of the order of $1000 \mathrm{~km}$. This corresponds to just a few $\mathrm{km}$ to a few tens of $\mathrm{km}$ when mapped to the ionosphere. Clearly at least an order of magnitude more is required, overlaps are often at least 0.1 degree (Norberg et al., 1994). This could possibly be provided by the more bent field lines resulting from subsolar reconnection. Such field-lines are not part of our data set and would require another study. The high end tail of our acceleration data corresponds to centrifugal acceleration well above $10 \mathrm{kms}^{-1}$, but one may still conclude that the inertial drift must be much higher in the high altitude cusp proper than in the mantle where our observations take place, if this mechanism is to explain overlapping injections.

\section{Conclusions}

We have shown that the centrifugal acceleration is an important energization mechanism for outflowing $\mathrm{O}^{+}$ions. The acceleration is often $10 \mathrm{~ms}^{-2}$ and frequently reaches $100 \mathrm{~ms}^{-2}$. If such net accelerations persist for several Earth radii travel distance along the field-line the resulting energy increase of an $\mathrm{O}^{+}$ion will be several tens to several $100 \mathrm{eV}$. At high altitude, reasonably close to the magnetopause, the acceleration is often even larger, $1 \mathrm{kms}^{-2}$ and above.

Dividing the centrifugal acceleration into its three terms, parallel, perpendicular and temporal, it is found that the perpendicular term provides more acceleration than the parallel term but they are close to each other and both are important. The temporal term is more important for the strongest accelerations, but produces approximately as much deceleration as acceleration. A rough estimate of the field-aligned velocity resulting from the observed centrifugal acceleration 
at altitudes below the observation point was performed for each orbit. The distribution of velocities obtained this way was very similar to the actually observed parallel velocities. This indicates that centrifugal acceleration can explain much of the observed field-aligned velocities of $\mathrm{O}^{+}$in the high altitude polar cap region. A number of features of the high altitude $\mathrm{O}^{+}$as described in Nilsson et al. (2006b) can not be explained by centrifugal acceleration (such as increasing perpendicular temperature with altitude), so other mechanisms also play a role. To determine the relative role of centrifugal acceleration requires that these mechanisms are also quantitatively estimated in the way we have done with the centrifugal acceleration in this study.

It was also investigated how much the estimates of the centrifugal acceleration were affected if the magnetic field was taken from the Tsyganenko T89 model instead of from the measurements. The model magnetic field results could not reproduce the highest accelerations, in particular for high altitudes corresponding to magnetic fields of less than $50 \mathrm{nT}$. For lower altitudes the agreement was fairly good with only minor, though systematic differences of the order of $10 \%$. Therefore much of the results reported here should be possible to reproduce using test particles in a model magnetic field with a realistic convection model and an initial velocity of $20 \mathrm{~km} \mathrm{~s}^{-1}$ at $5 R_{E}$. The latter value is the median of observed parallel velocities in the lowest altitude point of the ion beam data from each orbit.

We have estimated the inertial drift of the $\mathrm{O}^{+}$particles in the direction of the electric field which is associated with the centrifugal acceleration. This drift is relatively often 1-10\% of the convection velocity and may therefore be of some significance. Finally we suggested that the same mechanism could explain the overlapping cusp injections. A test of this suggestion should be possible to perform with a test particle code with a realistic magnetic field and convection model. However our initial estimate indicates that the inertial drift in the mantle where our observations were made was at least an order of magnitude too small to explain overlapping injections.

Acknowledgements. This work was supported by the National Space Board of Sweden and by the funding agencies of our coauthors. The $K_{p}$ index is distributed by WDC C2 for geomagnetism, Kyoto, Japan.

Topical Editor I. A. Daglis thanks M. Hirahara and another anonymous referee for their help in evaluating this paper.

\section{References}

Antonova, Y. Y.: Transverse acceleration of ionospheric ions induced by collisions with moving inhomogeneities, Geomag. Aeronomy, 23, 610-612, 1983.

Arvelius, S., Yamauchi, M., Nilsson, H., Lundin, R., Hobara, Y., Rème, H., Bavassano-Cattaneo, M. B., Paschmann, G., Korth, A., Kistler, L., and Parks, G. K.: Statistics of high-altitude and high-latitude $\mathrm{O}^{+}$ion outflows observed by Cluster/CIS, Ann.
Geophys., 23, 1909-1916, 2005,

http://www.ann-geophys.net/23/1909/2005/.

Balogh, A., Carr, C. M., Acuna, M. H., Dunlop, M. W., Beek, T. J., Brown, P., Fornaon, K.-H., Georgescu, E., Glassmeier, K.-H., Harris, J., Musmann, G., Oddy, T., and Schwingenschuh, K.: The Cluster Magnetic Field Investigation: overview of in-flight performance and initial results, Ann. Geophys., 19, 1207-1217, 2001 ,

http://www.ann-geophys.net/19/1207/2001/.

Bogdanova, Y. V., Fazakarley, A. N., Owen, C. J., Klecker, B., Cornilleau-Wehrlin, N., Grison, B., Andrẽ, M., Cargill, P., Rème, H., Bosqued, J. M., Kistler, L. M., and Balogh, A.: Correlation between suprathermal electron bursts, broadband extremely low frequency waves, and local ion heating in the midaltitude cleft/low-altitude boundary layer observed by Cluster, J. Geophys. Res., 109, A12226, doi.10.1029/2004JA010554, 2004.

Bouhram, M., Klecker, B., Miyake, W., Rème, H., Sauvaud, J.-A., Malingre, M., Kistler, L., and Blăgău, A.: On the altitude dependence of transversely heated $\mathrm{O}^{+}$distributions in the cusp/cleft, Ann. Geophys., 22, 1787-1798, 2004, http://www.ann-geophys.net/22/1787/2004/.

Chaston, C. C., Bonnell, J. W., Carlson, C. W., McFadden, J. P., Ergun, R. E., Strangeway, R. J., and Lund, E. J.: Auroral ion acceleration in dispersive Alfvén waves, J. Geophys. Res., 109, A04205, doi:10.1029/2003JA010053, 2004.

Cladis, J. B.: Parallel acceleration and transport of ions from polar ionosphere to plasmasheet, J. Geophys. Res., 13, 893-896, 1986.

Daglis, I. A. and Axford, I.: Fast ionospheric response to enhanced activity in geospace: Ion feeding of the inner magnetotail, J. Geophys. Res., 5047-5065, 1996.

Delcourt, D. C.: Centrifugal effects near the frontside magnetopause, J. Geophys. Res., 99, 8585-8591, 1994.

Delcourt, D. C., Moore, T. E., Orsini, S., Millilo, A., and Sauvaud, J.-A.: Centrifugal acceleration of ions near Mercury, Geophys. Res. Lett., 29, 32.1-32.4, 2002.

Demars, H. G., Barakat, A. R., and Schunk, R. W.: Effect of centrifugal acceleration on the polar wind, J. Geophys. Res., 101, 24 565-24 571, 1996.

Dubouloz, N., Bouhram, M., Senior, C., Delcourt, D., Malingre, M., and Sauvaud, J.-A.: Spatial structure of the cusp/cleft ion fountain: A case study using a magnetic conjugacy between Interball AP and a pair of SuperDARN radars, J. Geophys. Res., 106, 261-274, 2001.

Ebihara, Y., Yamada, M., Watanabe, S., and Ejiri, M.: Fate of upflowing suprathermal oxygen ions that originate in the polar ionosphere, J. Geophys. Res., 111, A04219, doi:10.1029/2005JA011403, 2006.

Eriksson, A. I., André, M., Klecker, B., Laakso, H., Lindqvist, P.A., Mozer, F., Paschmann, G., Pedersen, A., Quinn, J., Torbert, R., Torkar, K., and Vaith, H.: Electric field measurements on Cluster: comparing double-probe and electron drift techniques, Ann. Geophys., 24, 275-289, 2006, http://www.ann-geophys.net/24/275/2006/.

Frank, L. A., Ackerson, K. L., and Yeager, D. M.: Observations of atomic oxygen $\left(\mathrm{O}^{+}\right)$in the Earth's magnetotail, J. Geophys. Res., 82, 129-133, 1977.

Guglielmi, A. and Lundin, R.: Ponderomotive upward acceleration of ions by ion cyclotron and Alfvén waves over the polar regions, J. Geophys. Res., 106, 13 219-13 236, 2001. 
Haider, S. A.: High-latitude plasma transport through the Martian tail: Polar wind, J. Geophys. Res., 101, 24 955-24 963, 1996.

Harvey, C. C.: Analysis Methods for Multi-spacecraft data, chap. 12, pp. 307-322, ISSI Scientific report SR-001, ESA Publications Division, 1998.

Hirahara, M., Mukai, T., Terasawa, T., Machida, S., Saito, Y., Yamamoto, T., and Kokubun, S.: Cold dense ion flows with multiple components observed in the distant tail lobe by Geotail, J. Geophys. Res., 101, 7769-7784, 1996.

Horwitz, J. L., Ho, C. W., Scarbro, H. D., Wilson, G. R., and Moore, T. E.: Centrifugal acceleration of the polar wind, J. Geophys. Res., 99, 15 051-15 064, 1994.

Korth, A. and Pu, Z. Y.: Magnetic field configuration and fieldaligned acceleration of energetic ions during substorm onsets, Ann. Geophys., 19, 1089-1094, 2001, http://www.ann-geophys.net/19/1089/2001/.

Liu, C., Perez, J. D., Moore, T. E., and Chappell, C. R.: Low energy particle signature of substorm dipolarization, Geophys. Res. Lett., 21, 229-232, 1994.

Nilsson, H., Yamauchi, M., Eliasson, L., Norberg, O., and Clemmons, J.: The ionospheric signature of the cusp as seen by incoherent scatter radar, J. Geophys. Res., 101, 10 947-10 963, 1996.

Nilsson, H., Joko, S., Lundin, R., Rème, H., Sauvaud, J.-A., Dandouras, I., Balogh, A., Carr, C., Kistler, L. M., Klecker, B., Carlson, C. W., Bavassano-Cattaneo, M. B., and Korth, A.: The structure of high altitude $\mathrm{O}^{+}$energization and outflow: a case study, Ann. Geophys., 22, 2497-2506, 2004, http://www.ann-geophys.net/22/2497/2004/.

Nilsson, H., Carlsson, E., Gunell, H., Futaana, Y., Barabash, S., Lundin, R., Fedorov, A., Soobiah, Y., Coates, A., Fränz, M., and Roussos, E.: Investigation of the influence of magnetic anomalies on ion distributions at Mars, Space Sci. Rev., http: //dx.doi.org/10.1007/s11214--006--9030--0, 2006a.

Nilsson, H., Waara, M., Arvelius, S., Marghitu, O., Bouhram, M., Hobara, Y., Yamauchi, M., Lundin, R., Rème, H., Sauvaud, J.A., Dandouras, I., Balogh, A., Kistler, L. M., Klecker, B., Carlson, C. W., Bavassano-Cattaneo, M. B., and Korth, A.: Characteristics of high altitude oxygen ion energization and outflow as observed by Cluster; a statistical study, Ann. Geophys., 24, 1099-1112, 2006b.

Norberg, O., Yamauchi, M., Eliasson, L., and Lundin, R.: Freja observations of multiple injection events in the cusp, Geophys. Res. Lett., 21, 1919-1922, 1994.

Northrop, T. G.: The adiabatic motion of charged particles, Interscience Publishers, N.Y., 1963.
Rème, H., Aoustin, C., Bosqued, J. M., Dandouras, I., Lavraud, B., Sauvaud, J. A., Barthe, A., Bouyssou, J., Camus, T., Coeur-Joly, O., Cros, A., Cuvilo, J., Ducay, F., Garbarowitz, Y., Medale, J. L., Penou, E., Perrier, H., Romefort, D., Rouzaud, J., Vallat, C., AlcaydE, D., Jacquey, C., Mazelle, C., d'Uston, C., Möbius, E., Kistler, L. M., Crocker, K., Granoff, M., Mouikis, C., Popecki, M., Vosbury, M., Klecker, B., Hovestadt, D., Kucharek, H., Kuenneth, E., Paschmann, G., Scholer, M., Sckopke, N., Seidenschwang, E., Carlson, C. W., Curtis, D. W., Ingraham, C., Lin, R. P., McFadden, J. P., Parks, G. K., Phan, T., Formisano, V., Amata, E., Bavassano-Cattaneo, M. B., Baldetti, P., Bruno, R., Chionchio, G., Lellis, A. D., Marcucci, M. F., Pallocchia, G., Korth, A., Daly, P. W., Graeve, B., Rosenbauer, H., Vasyliunas, V., McCarthy, M., Wilber, M., Eliasson, L., Lundin, R., Olsen, S., Shelley, E. G., Fuselier, S., Ghielmetti, A. G., Lennartsson, W., Escoubet, C. P., Balsiger, H., Friedel, R., Cao, J.-B., Kovrazhkin, R. A., Papamastorakis, I., Pellat, R., Scudder, J., , and Sonnerup, B.: First multispacecraft ion measurements in and near the Earth's magnetosphere with the identical Cluster ion spectrometry (CIS) experiment, Ann. Geophys., 19, 1303-1354, 2001, http://www.ann-geophys.net/19/1303/2001/.

Seki, K., Hirahara, M., Terasawa, T., Mukai, T., Saito, Y., Machida, S., Yamamoto, T., and Kokubun, S.: Statistical properties and possible supply mechanisms of tailward cold $\mathrm{O}^{+}$beams in the lobe/mantle regions, J. Geophys. Res., 103, 4477-4489, 1998.

Swift, D. W.: Simulation of the ejection of plasma from the polar ionosphere, J. Geophys. Res., 95, 12103 - 12 118, 1990.

Torkar, K., Fehringer, M., Escoubet, C. P., André, M., Pedersen, A., Svenes, K. R., and Décréau, P. M. E.: Analysis of Cluster spacecraft potential during active control, Adv. Space. Res., 36, 1922-1927, 2005.

Tsyganenko, N. A.: A magnetospheric model with a warped tail current, Plan. Space Sci., 37, 5-20, 1989.

Winglee, R. M.: Ion cyclotron and heavy ion effects on reconnection in a global magnetotail, J. Geophys. res., 109, doi:10.1029/2004JA010385, 2004.

Winglee, R. M., Chua, D., Brittnacher, M., Parks, G. K., and Lu, G.: Global impact of ionospheric outflows on the dynamics of the magnetosphere and cross-polar cap potential, J. Geophys. Res., 107, 1237, doi:10.1029/2001JA000214, 2002.

Yamauchi, M. and Lundin, R.: Comparison of various cusp models with high- and low-resolution observations, Space Sci. Rev., 95, 457-468, 2001. 